\title{
Postural Orthostatic Tachycardia Syndrome
}

National Institute of Neurological Disorders and Stroke (NINDS)

\section{Source}

National Institute of Neurological Disorders and Stroke (NINDS). Postural Tachycardia Syndrome Information Page.

Postural orthostatic tachycardia syndrome (POTS) is one of a group of disorders that have orthostatic intolerance $(\mathrm{OI})$ as their primary symptom. OI describes a condition in which an excessively reduced volume of blood returns to the heart after an individual stands up from a lying down position. The primary symptom of OI is lightheadedness or fainting. In POTS, the lightheadedness or fainting is also accompanied by a rapid increase in heartbeat of more than 30 beats per minute, or a heart rate that exceeds 120 beats per minute, within 10 minutes of rising. The faintness or lightheadedness of POTS are relieved by lying down again. Anyone at any age can develop POTS, but the majority of individuals affected (between 75 and 80 percent) are women between the ages of 15 to 50 years of age. Some women report an increase in episodes of POTS right before their menstrual periods. POTS often begins after a pregnancy, major surgery, trauma, or a viral illness. It may make individuals unable to exercise because the activity brings on fainting spells or dizziness.

Doctors aren't sure yet what causes the reduced return of blood to the heart that occurs in Ol, or why the heart beg ins to beat so rapidly in POTS. Current thinking is that there are a number of mechanisms. Some individuals have peripheral denervation (neuropathic POTS); some have symptoms that are due to sustained or parosyxmal overactivity of the sympathetic nervous system (hyperadrenergic POTS); and many individuals with POTS have significant deconditioning. 